\title{
Identification of fatal outcome in a childhood nasopharyngeal carcinoma patient by protein expression profiling
}

\author{
MOHAMED E.M.SAEED ${ }^{1}$, ROLF MERTENS ${ }^{2}$, RUPERT HANDGRETINGER $^{3}$ and THOMAS EFFERTH $^{1}$ \\ ${ }^{1}$ Department of Pharmaceutical Biology, Institute of Pharmacy and Biochemistry, Johannes Gutenberg University, \\ D-55128 Mainz; ${ }^{2}$ Section for Pediatric Hematology, Oncology and Stem Cell Transplantation, \\ Department of Pediatric and Adolescent Medicine, University Hospital Aachen, D-52047 Aachen; \\ ${ }^{3}$ Department of Paediatric Haematology/Oncology, Children's University Hospital, D-72076 Tübingen, Germany
}

Received December 12, 2017; Accepted March 29, 2018

DOI: $10.3892 /$ ijo.2018.4491

\begin{abstract}
Nasopharyngeal carcinoma (NPC) is a rare disease in children with good prognosis and high cure rate. Nevertheless, certain patients have an unfavorable prognosis due to development of refractory NPC that is unresponsive to any therapeutic strategies. The current study studies a case of a 17 years-old female with non-keratinizing NPC type IIb (T2N0M0), who passed away as a consequence of resistance to chemo-, radio- and $\beta$-interferon therapy, and to an allogenic stem cell transplantation. In order to identify factors that lead to treatment failure and fatal outcome, immunohistochemical analyses of different tumor biomarkers and hierarchical cluster analysis were performed and compared with those of eight other patients with NPC who experienced complete remission following conventional therapy. Hierarchical cluster analysis of the immunohistochemical results clearly demonstrated that staining for immunological factors (CD4, CD8 and CD56) distinguished this patient from the others. To further investigate a potential role of the immune system, lymphocytic infiltration was assessed in tumor tissue by evaluation of hematoxylin and eosin-stained tumor sections. Indeed, no
\end{abstract}

Correspondence to: Professor Thomas Efferth, Department of Pharmaceutical Biology, Institute of Pharmacy and Biochemistry, Johannes Gutenberg University, Staudinger Weg 5, D-55128 Mainz, Germany

E-mail: efferth@uni-mainz.de

Abbreviations: AAC, ATP-ADP translocase; CTL, cytotoxic T-lymphocyte; EBV, Epstein-Barr virus; eIF3, eukaryotic translation initiation factor 3; GSTP1, glutathione S-transferase pi 1; $\mathrm{H} \& \mathrm{E}$, hematoxylin and eosin; HHV-6, human herpes virus 6; HPV, human papilloma virus; HSV, herpes simplex virus; iNOS, inducible nitric oxide synthetase; INF, interferon; NK, natural killer; NPC, nasopharyngeal carcinoma; TIL, tumor-infiltrating lymphocyte; Tregs, T-regulatory cells; WHO, world health organization; VZV, Varicella zoster virus; WT1, Wilms tumor 1

Key words: biomarker, hierarchical cluster analysis, pediatric cancer, tumor-infiltrating lymphocytes tumor infiltrating lymphocytes (TILs) were observed in this NPC case, while 7 out of 8 of the other NPC samples contained variable TIL amounts. The view that immunodeficiency of the patient may be a factor in the fatal outcome of treatment is supported by the fact that this patient with NPC was not positive for Epstein-Barr virus markers and also infected by several other viruses and fungi (herpes simplex virus, human herpes virus 6, Varicella zoster virus, and Candida). In conclusion, the investigation of rare NPC cases with poor prognosis may provide an improved understanding of the molecular mechanisms involved in refractory tumors and identification of novel potential therapeutic targets for NPC in the future.

\section{Introduction}

Nasopharyngeal carcinoma (NPC) is a tumor that arises from the epithelial lining of the nasopharynx, and it is considered the most common cancer of the head and neck (1). NPC has a distinct racial, sex and geographical distribution with a multifactorial etiology. China, Southeast Asia and North Africa have the highest prevalence worldwide. According to World Health Organization (WHO) estimates, the Western Pacific and Southeast Asia regions accounted for $\sim 77 \%$ of all cases internationally. Furthermore, NPC is particularly more frequent in males (sex ratio 2.3:1) and certain ethnicities, such as Chinese $(2,3)$. The occurrence of NPC in childhood is rare. It has been estimated that $5 \%$ of childhood primary tumors arise in the head and neck area, while NPC only constitutes $2 \%$ of head and neck tumors in children $(4,5)$.

Multiple etiological factors have been reported to be as associated with the risk of developing of NPC, including genetics [certain human leukocyte antigen (HLA) types], environmental factors (nitrosamine and tobacco) and viral infection [Epstein Barr virus (EBV)] $(6,7)$. The WHO has classified the NPC into three types, based on histopathological criteria: Keratinizing squamous cell carcinoma (type 1), non-keratinizing carcinoma (type 2) and undifferentiated carcinoma (type 3). It has been reported that these types are geographically diversified. In Southern China, where NPC is highly prevalent, nearly all cases are of the undifferentiated type, whereas in the USA, where NPC is rare, $20 \%$ cases are the keratinizing type (8). 
Although EBV has long been associated with nasopharyngeal carcinogenesis, several studies have reported an association of oncogenic human papillomaviruses (HPV) with a sub-group of NPCs (9-12). Furthermore, HPV is considered as a leading cause of cervical carcinoma (13). Whilst HPV has been reported as one of the causative agents of NPC in adults, little is known about the implication of HPV in the pathogenesis of childhood NPC.

In the present study, a rare case of a patient with a low-risk NPC type IIb with T2NOM0, which was expected to have a good prognosis, is reported. Surprisingly, the tumor was refractory to therapy and even allogenic stem cell transplantation failed, which ultimately led to mortality. The poor prognosis was not predictable by standard clinicopathological parameters. Thus, the expression of a panel of biomarkers was analyzed by immunohistochemistry in formalin-fixed, paraffin-embedded (FFPE) tumor material from this patient and compared with biopsies from 8 other childhood NPC cases. All 9 patients were enrolled in the prospective multicenter study [Nasopharyngeal Carcinoma (NPC) 2003 German Society of Pediatric Oncology and Hematology/German Children's Oncology Group (NPC-2003-GPOH/DCOG)] (14,15). The biomarkers included human papilloma virus markers (E6, E7), immunological markers (CD4, CD8 and CD56), proliferation markers [Ki-67, 14-3-3 $\tau$, eukaryotic translation initiation factor 3 subunit E (eIF3e)], tumor suppressors [p53, Wilms tumor 1 (WT1)], apoptosis markers [terminal deoxynucleotidyl transferase dUTP nick-end labeling (TUNEL) assay, p53, ATP-ADP translocase (AAC)] and other markers [glutathione S-transferase $\pi 1$ (GSTP1), CD31, inducible nitric oxide synthetase (iNOS)].

\section{Materials and methods}

Patients. A 17 year-old female patient was diagnosed with a low-risk nasopharyngeal tumor at the Department for Pediatrics (Medical Center of the University of Tübingen, Tübingen, Germany) on March 16th, 2007. The histology was determined as non-keratinizing squamous epithelial carcinoma with lymphoid infiltration of type IIb with T2N0M0 stage. Therapy was performed according to NPC-2003-GPOH (14) and consisted of: i) Tumor irradiation including lymph vessels with 59.4 and 45 Gy, respectively; ii) two chemotherapy cycles with cisplatin concomitant to irradiation during the first cycle and carboplatin during the second cycle (because of tinnitus as side effect); and iii) $\beta$-interferon ( $\beta$-IFN).

A systemically refractory tumor was diagnosed on February 7 th, 2008. The patient suffered from severe pain symptoms and atelectasis pneumonia of the left lower lobe. Chemotherapy was started according to NPC-2003-GPOH with cisplatin and 5-fluorouracil in the first cycle and cisplatin, 5 -fluorouracil and docetaxel in the second cycle.

A second refractory tumor was diagnosed on October 17th, 2008. The patient was treated with chemotherapy again (cisplatin, 5-fluorouracil and docetaxel). Recurrence was diagnosed on February 7th, 2008. Irradiation could not be performed, because of the large expansion of the tumor (intra-abdominal, retro-crural, intra-thoracic, along the Truncus coeliacus and para-aortic). AS chemotherapy alone was not curative, allogenic stem cell transplantation was suggested. In parallel, there were signs of immunodeficiency, indicated by infection with Herpes simplex virus (HSV), human-HSV-6 (HHV-6), Varicella zoster virus (VZV) and Candida ssp. in addition to EBV. Thus, allogenic stem cell transplantation from the HLA-identical 9 year-old brother of the patient was suggested as a potential curative treatment approach. As the brother was EBV-IgG-positive, it was expected that a T-cell-mediated antiviral response against EBV may be transferred by transplantation. The patient's tumor cells expressed EBV antigens. Therefore, an antiviral T-cell response may act as allo-versustumor response and, thus, as prophylaxis against renewed recurrence of the tumor.

Following agreement of the patient, the stem cell transplantation was performed. Conditioning took place at June 4th, 2008 with treosulfan and cyclophosphamide and was well tolerated except for a transient increase of liver transaminases and nausea. The allogenic bone marrow stem cells transferred on June 10th, 2008 contained $1.25 \times 10^{8}$ mononuclear cells $/ \mathrm{kg}$ body weight (BW), $2.45 \times 10^{6} \mathrm{CD} 34$-positive progenitor cells $/ \mathrm{kg}$ $\mathrm{BW}, 0.70 \times 10^{6} \mathrm{AC} 133$-positive progenitor cells $/ \mathrm{kg} \mathrm{BW}$ and $1,661.43 \times 10^{4} \mathrm{CD} 3$-positive cells/kg BW. Antibiotic prophylaxis was performed with metronidazole $(800 \mathrm{mg} / \mathrm{day}$ for 29 days) and cotrimoxazol (160 mg/day for 28 days), fungal prophylaxis with amphotericin B (150 mg/day for 29 days) and viral prophylaxis with acyclovir (2,400 mg/day for 35 days). Graft-versus-host disease was treated with cyclosporine $(90 \mathrm{mg} /$ day for 16 days), methotrexate $(15 \mathrm{mg} /$ day for 1 day) and prednisolone (100 mg/day for 5 days). Granulocyte colony stimulating factor growth factor was applied 11 times between day 4 and 14 after transplantation. The course of the transplantation was free of complications.

Toxicity (WHO grade IV) occurred in the aplastic phase (mucositis, nausea, transient increase of transaminases). Graft-versus-host disease of the skin (grade I) appeared on day 13 after transplantation. The engraftment took place on June 24th, 2008 (day 14 after transplantation). Arterial hypertension was observed as side effect of drug therapy. The atelectasis of the left lower lobe was persisting. Recurrence following stem cell transplantation was diagnosed on October 17th, 2009 and the patient died on January 25th, 2009.

In addition to this problematic patient, 8 pediatric NPC patients (age range, 11-17 years at diagnosis; 5:3 male:female ratio) that achieved complete remission following treatment according to the NPC-2003-GPOH regimen were investigated. FFPE tumor biopsies from all patients were provided by Dr Rolf Mertens (University Hospital Aachen, Aachen, Germany) and analyzed for comparison with the aforementioned clinical case. The tumor tissues were fixed in $10 \%$ neutral-buffered formalin at room temperature for $24 \mathrm{~h}$. These FFPE samples have been investigated within the frame of the NPC-2003-GPOH/DCOG study (14).

Ethical approval (reference no. EK034/03) for the use of tumor material for experimental purposes was obtained from the Ethics Committee of the University of Aachen (Aachen, Germany), and local ethical approval was obtained from the participating centers in Germany (14). Written informed consent was obtained from all patients.

Immunohistochemistry staining and evaluation. Commercially available antibodies were applied on paraffin-embedded tissue 
slides using a polymeric labeling technique. Briefly, all slides were washed twice with xylene $(98.5 \%$ xylene for 5 mineach wash at room temperature) to remove paraffin. Primary antibodies used in immunohistochemistry staining were: iNOS (1:150; clone K13-A; cat. no. DB 003-0.05; Acris Antibodies; OriGene Technologies, Inc., Rockville, MD, USA), p53 (1:100; clone Y5; cat. no. MA5-14467; Thermo Fisher Scientific, Inc., Waltham, MA, USA), Ki-67 (1:200; clone SP6; cat. no. ab16667; Abcam, Cambridge, UK), c-Myc (1:50; clone 9E10.3; cat. no. AHO0062), CD31 (1:150; clone JC/70A; cat. no. MA5-13188), CD56 (1:100; clone 56C04; cat. no. MA5-11563), CD8 (1:50; clone SP16; cat. no. MA5-14548), CD4 (1:10; clone 4B12; cat. no. MA5-12259) (all from Thermo Fisher Scientific, Inc.), AAC (1:50 polyclonal; cat. no. 51031-1-AP; ProteinTech Group, Inc., Chicago, IL, USA), GSTP1 (1:1,500; clone 3F2C2; cat. no. LS-B1576-50; Acris Antibodies; OriGene Technologies, Inc.), WT1 (1:10; clone 6F-H2; cat. no. MA1-46028; Thermo Fisher Scientific,

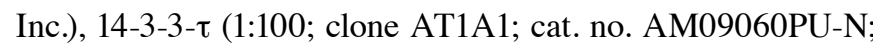
Acris Antibodies; OriGene Technologies, Inc.), E6 (1:50; clone C1P5; cat. no. MA1-46057; Thermo Fisher Scientific, Inc.) and E7 (1:100; clone TVG701Y; cat. no. MA5-14132; Thermo Fisher Scientific, Inc.). The TUNEL assay used was Apoptag ${ }^{\circledR}$ Peroxidase Detection Kit (EMD Millipore, Billerica, MA, USA). Then, sample tissues were rehydrated through graded washes with isopropanol in water (starting from $99 \%$ to $70 \%$ isopropanol for $1 \mathrm{~min}$ in each wash at room temperature). Heat-induced epitope retrieval was performed using a pressure cooker $\left(100^{\circ} \mathrm{C}\right.$ for $\left.20 \mathrm{~min}\right)$ as a heating device. Retrieval solutions were used ( $1 \%$ citrate buffer $\mathrm{pH} 6$ in $\mathrm{PBS})$, then cooling was performed on ice for $25 \mathrm{~min} . \mathrm{H}_{2} \mathrm{O}_{2}(3 \%)$ and Ultra-vision protein block (cat. no. TA-060-UB; Thermo Fisher Scientific, Inc.) were added for $10 \mathrm{~min}$ in each solution at room temperature to block endogenous peroxidase and avoid non-specific background staining. Slides were incubated overnight at $4^{\circ} \mathrm{C}$ following addition of primary antibodies, and then horseradish peroxidase-labeled polymers conjugated with secondary antibodies (cat. no. TL-060-QPH; Thermo Fisher Scientific, Inc.) were added. The final staining reaction was performed with diaminobenzidine and slides were counterstained with hematoxylin (cat. nos. TA-060-QHSX and TA-002-QHCX; Thermo Fisher Scientific, Inc.). All solutions used for blocking and detection were provided from Thermo Fisher Scientific, Inc. (UltraVision Quanto Detection System HRP DAB; cat. no. TL-060-QHD) and the protocol was followed according to the manufacturer's instructions. The immunostained slides were scanned by Panoramic Desk and quantified by Panoramic Viewer software version 1.15.4 (NuclearQuant and MembraneQuant) (both from 3DHISTECH Ltd., Budapest, Hungary). Quantification (percentage of positivity) was calculated by dividing the number of positively stained cells by an overall number of cells found each six independently annotated tumor areas. Mean values revealed the average protein expression, while standard deviation values indicated the heterogeneity of expression in the tissue. Higher standard deviation indicated higher heterogeneity of expression of the corresponding protein.

Assessment of tumor-infiltrating lymphocytes (TILs). Histological hematoxylin and eosin (H\&E) routine staining (16) was performed on tumor sections, in order to assess TILs. The cells were counted with panoramic viewer software (NuclearQuant; 3DHISTECH Ltd.) according to the recommendations of the International TILs Working Group 2015 (17): i) The percentage of TILs in the stromal tumor tissue within the borders of the invasive tumor has been determined. TILs in normal areas outside the tumor were not considered; ii) tumor areas with crush artifacts, necrosis, or regressive hyalinization were not evaluated; iii) only mononuclear, but not polymorphonuclear leukocytes were counted; iv) the average values of the full tumor area were assesses, rather than focusing on hotspots.

Hierarchical cluster analysis. In the present study, hierarchical cluster analysis was performed to group heterogeneous objects into clusters of homogeneous objects. All objects were assembled into a cluster tree (dendrogram). Thus, objects with tightly related features appear together, whereas the separation in the cluster tree increases with progressive dissimilarity. The merging of objects with similar features leads to formation of a cluster, the shortest the distance of the branch the closest degree of relatedness (18). Hierarchical clustering was conducted using WinSTAT software (R. Fitch Software, Cambridge, MA, USA) and the Ward method (19) was performed. Previously, cluster methods have been validated for gene expression profiling, identification of candidate genes for drug resistance and sensitivity and for understanding molecular biology of cancer $(20,21)$.

\section{Results}

Immunohistochemistry. An expression of a panel of biomarkers for tumor aggressiveness and progression was analyzed in a female patient with NPC type IIb (case 9). The expression patterns were compared with those of eight other patients with NPC (cases 1 to 8). Using immunohistochemistry, 15 different markers were detected, which may indicate tumor aggressiveness and may also predict tumor responsiveness to therapy. Representative immunostaining images of NPC case 9 and selected tumors of the other patients with NPC are depicted in Figs. 1-3; staining for human papilloma virus markers (E6, E7; Fig. 1), immunological markers (CD4,

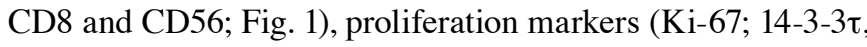
eIF3e; Fig. 2), tumor suppressors (p53, WT1; Fig. 2), apoptosis markers (TUNEL assay, p53, AAC; Fig. 3) and other markers (GSTP1, CD31, iNOS; Fig. 3) is shown.

A general observation in most tumor tissues was that the expression pattern exhibited considerable heterogeneity; the number of stained cells and the staining intensities varied between different areas of the same tumor. For this reason, the immunostaining for all antibody and all tumors was quantified. Six representative areas were chosen from each tumor and the number of positively stained cells was counted using a digital slide scanning (Fig. 4).

Hierarchical cluster analysis. This technique is a widely used methodology to extract relevant results from transcriptomic data sets. It uses a distance/proximity-based approach to identify structures in large data sets. In systems biology, cluster analyses are valuable to define profiles of genes ('gene signatures'), whose expression is linked to biological phenomena, i.e. histological 


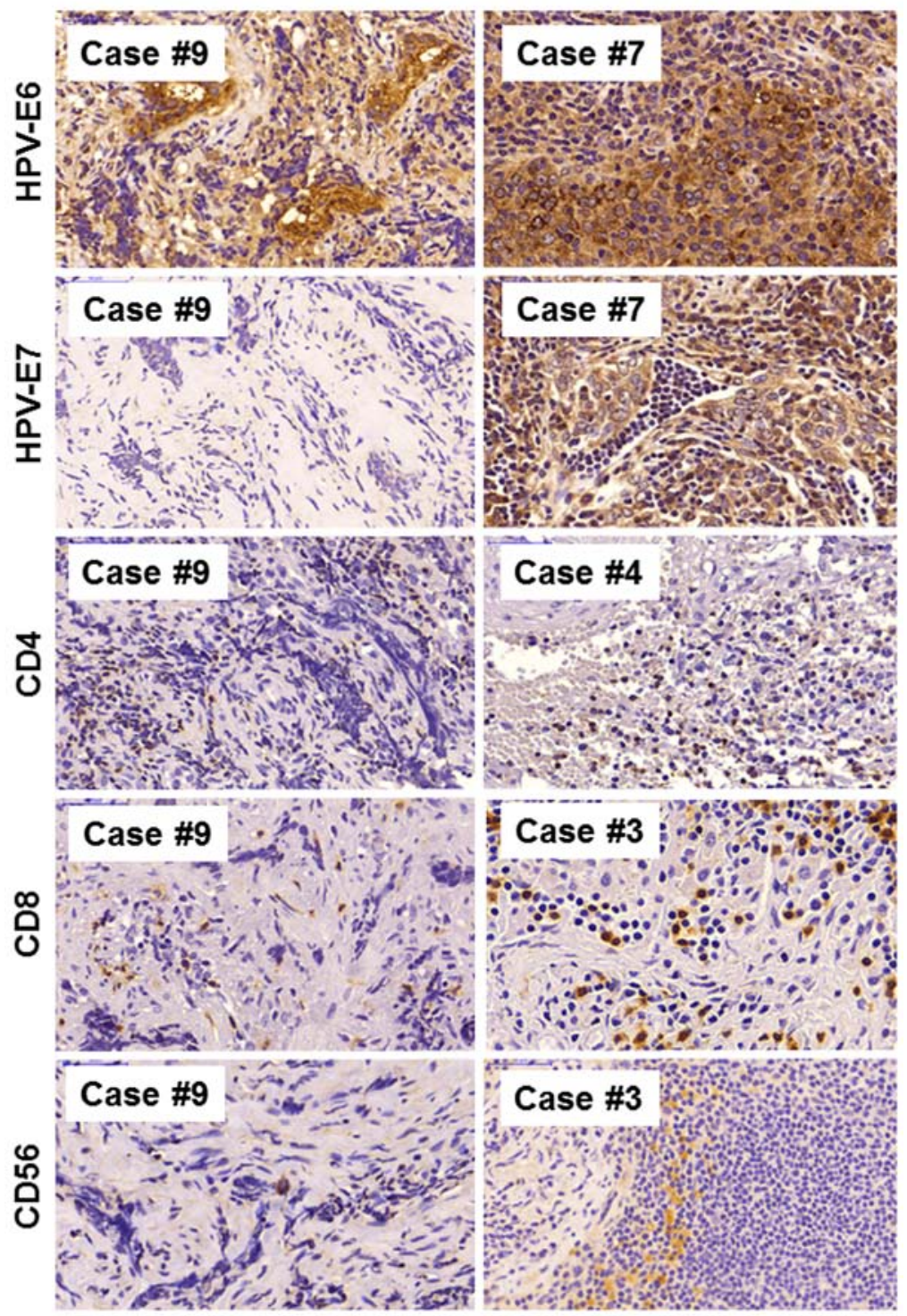

Figure 1. Representative immunohistochemical staining for viral markers (HPV-E6 and E7) and immunological markers (CD4, CD8 and CD56) in pediatric nasopharyngeal carcinoma. HPV-E6 and HPV-E7 were expressed in the tumor cells, while CD4, CD8 and CD56 were expressed in tumor-infiltrating lymphocytes. Magnification, $\mathrm{x} 40$. HPV, human papilloma virus.

subtypes of tumors, resistance or sensitivity of tumors towards anticancer treatments, survival chances of cancer patients, etc. Among the numerous clustering methods (e.g. topological interaction models, influence maps, physical regulatory maps, self-organizing maps, principal component analysis, etc.), supervised, hierarchical and aggregative techniques provide advantages for pharmacological questions in cancer biology and pharmacology, because of their flexibility, possibility to include biological knowledge with different weighting, and detection of higher-order relationships between clusters of profiles (22). Aggregative hierarchical clustering is a frequently used method to investigate gene expression signatures (23-25).

The quantified expression values obtained from immunohistochemistry were subjected to hierarchical cluster analysis in order to investigate, whether the protein expression profile of patient 9 , who died following standard therapy and allogenic stem cell transplantation, could be differentiated from the profiles of the eight other NPC samples from patients that responded well to treatment.
If the results of all immunostainings were subjected to cluster analysis, a dendrogram with two clearly distinguishable branches appeared (Fig. 5A). However, case 9 did not appear as isolated branch separated from all other tumors, indicating that the panel of immunohistochemical parameters in its entirety was not able to identify this tumor.

Additionally, immunohistochemical staining markers were divided into groups consisting of immunological parameters [CD4 and CD8 as T-cell markers, and CD56 expression as marker of natural killer (NK) cells] and the remaining biomarkers were non-immunological markers. As shown in Fig. 5B and C, case 9 appeared isolated as single tumor in the dendrogram of the three immunological markers. The CD counts in case 9 were not the lowest of all samples, but the intermediate to low expression was different from the expression profile of these markers in the other NPC cases. Thus, cluster analysis separated case 9 as being distinct from the others when stained with immunological markers. The immunodeficient state of the patient was also validated by 

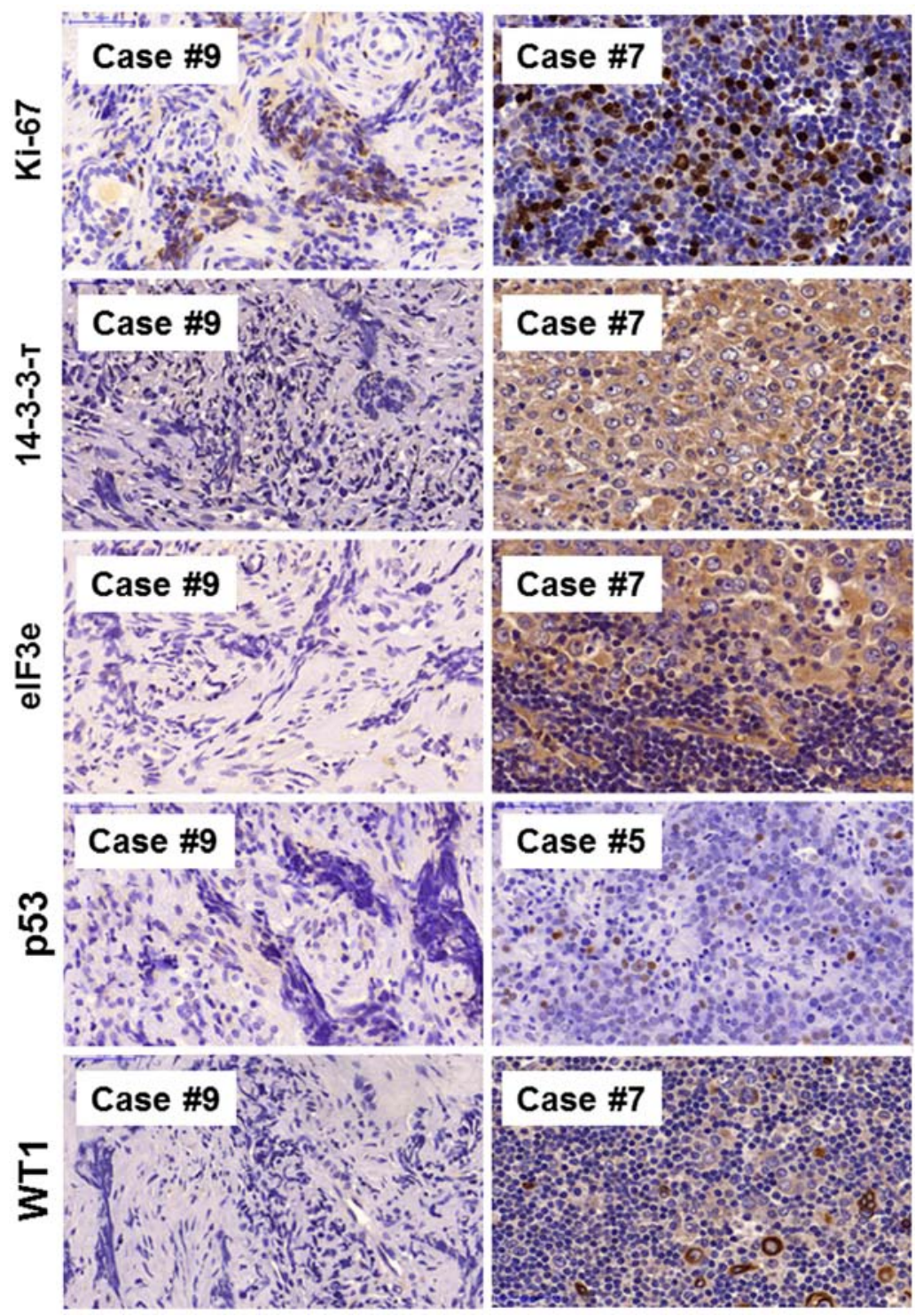

Figure 2. Representative immunohistochemical staining for proliferation markers (Ki-67, 14-3-3- $\tau$ and eIF3e) and tumor suppressors (p53 and WT1) in pediatric NPC. Ki-67 and p53 were predominately expressed in the nucleus of NPC cells, while 14-3-3- $\tau$ and eIF3e showed cytoplasmic staining. Magnification, $x 40$. NPC, nasopharyngeal carcinoma; eIF3e, eukaryotic translation initiation factor 3 subunit E; WT1, Wilms tumor 1.

H\&E-staining of TILs, where the lack of TILs in this patient became apparent. This was not observed in the dendrogram of all other non-immunological markers, indicating a potential specific role of the immune response in patient 9 , which may differ from the other patients with NPC.

Assessment of TILs. To further validate that a deficient immune response may have contributed to the treatment outcome of NPC case 9, the percentage of TILs in tumor samples was evaluated using H\&E-staining. TILs were redstained and were counted with the same microscopic slide scanning procedure as used for immunohistochemistry, with the exception that NuclearQuant software was used for counting red-stained cells, rather than brown-stained cells. The bar chart in Fig. 6A shows that NPC case 9 and another tumor (case 8) did not contain TILs at all, whereas the other NPC cases contained variable fractions of TILs ranging from $0.06 \pm 0.05$ to $43.7 \pm 15.3 \%$. To further validate the specificity of TIL-staining by H\&E, serial NPC sections stained with CD4 or CD8 antibodies were compared. As revealed by staining of serial sections of case 3 tumor, the TIL population identified by H\&E-staining was also CD4- and CD8-positive in immunohistochemistry (Fig. 6B-D).

\section{Discussion}

NPC is a complex disease associated with EBV infection, environmental factors, and genetic aberrations (26). Although childhood NPC generally has a good prognosis, certain patients do not benefit from chemo- and radiotherapy (27). Although $\beta$-IFN-containing therapy regimens improve outcomes $(13,14)$, cure rates of $100 \%$ are still achieved. The current study presents a case of a 17 year-old patient that could not be cured and finally died from the disease. This was surprising, as the patient was diagnosed with a low-risk type IIb tumor (T2NOM0), which was expected to have a good prognosis. Cases like these are still enigmatic, as treatment outcome or prognosis cannot be predicted by established 

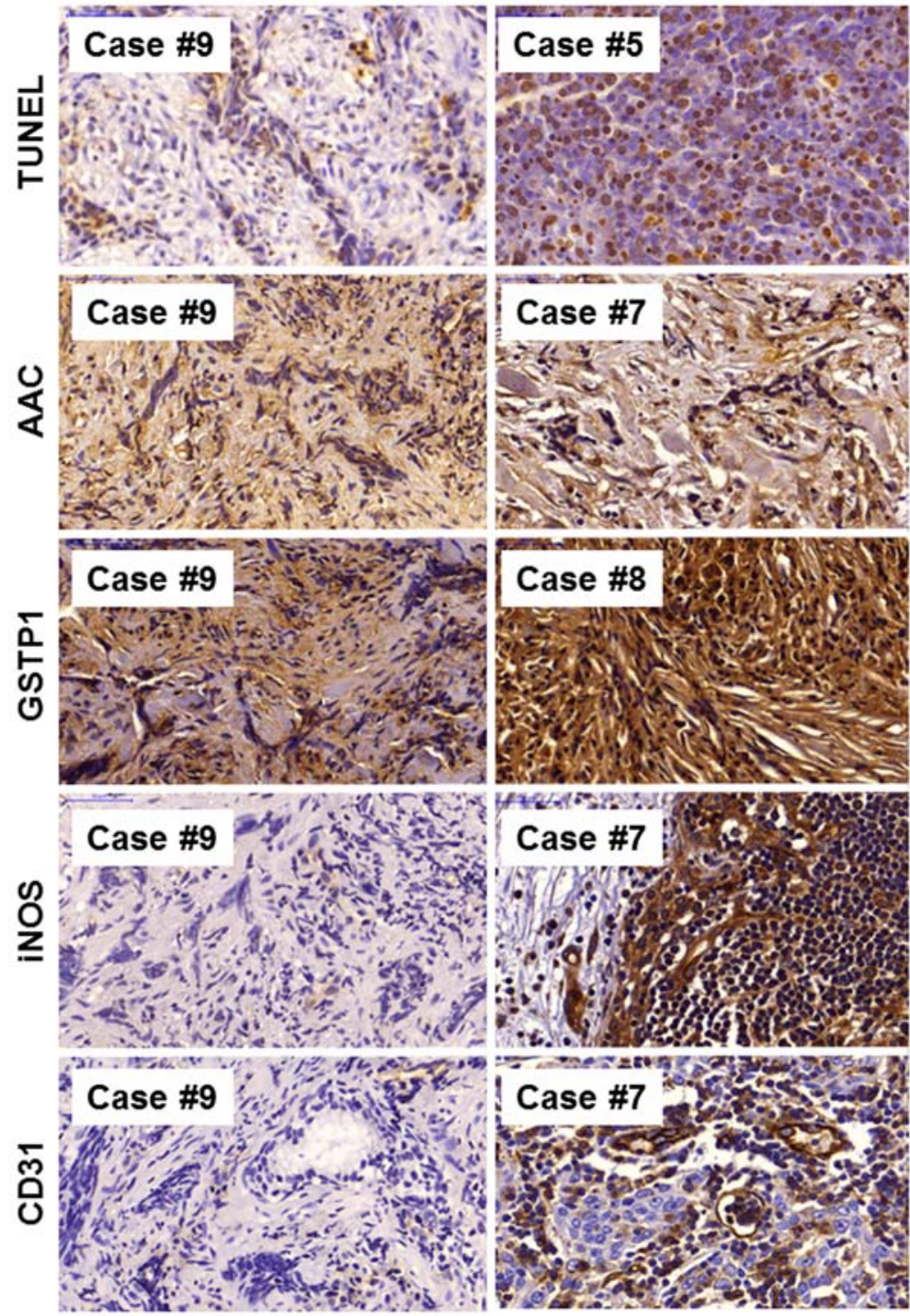

Figure 3. Representative immunohistochemical stainings for apoptotic markers (TUNEL assay, AAC) and other markers (GSTP1, iNOS, CD31) in pediatric nasopharyngeal carcinoma. The TUNEL assay stained the nuclei of apoptotic cells. AAC, GSTP1 and iNOS immunostaining was localized in the cytoplasm. CD31 stained tumor blood vessels and weak CD31 immunostaining was also observed outside the vessels in tumor cells. Magnification, x40. TUNEL, terminal deoxynucleotidyl transferase dUTP nick-end labelling; AAC, ATP-ADP translocase; GSTP1, glutathione S-transferase $\pi$ 1; iNOS, inducible nitric oxide synthetase.

clinical or pathoclinical parameters. Therefore, such cases deserve further attention and investigation.

To identify biomarkers that can predict rare cases of treatment non-responders, we immunohistochemical analyses were performed using a broad panel of antibodies against proteins involved in diverse cancer- and NPC-associated functions, including HPV, immune response, proliferation, tumor suppression, apoptosis and others. The quantified results were subjected to hierarchical cluster analysis, which is valuable for microarray-based mRNA-expression profiling, and also for protein expression data (28-30). The combination of three markers of immunological responses (CD4, CD8 and CD56) provided better separation of case 9 than when all markers were included.

CD4 and CD8 are key for the adoptive T-cell immune response. CD56 is a marker of NK cells, which are important for the innate immune system. Both categories of immune responses are involved in NPC. $\mathrm{CD}^{+}$and $\mathrm{CD} 8^{+} \mathrm{T}$-cells (TILs) infiltrate NPC $(28,29)$, and $\mathrm{CD} 56^{+} \mathrm{NK}$ cells act against
EBV-infected cells (31). Animal experiments demonstrated that CD8-positive T-cells enhance immunity to EBV-associated malignancies; $\mathrm{CD}^{+}$and $\mathrm{CD}^{+}{ }^{+}$T-cells act together synergistically in the antitumor response (32). In adult patients with NPC, it has been reported they have reduced numbers and function of cytotoxic T lymphocytes (CTLs) compared with normal subjects or long-term NPC survivors (33). In those patients, the cytolytic effects of NK cells were higher than that of healthy donors and NPC survivors (33). CTLs and NK cells compensate each other and are involved in immunity against NPC (33).

Innate and adaptive immunity may exert antitumor responses in some, but not all patients with tumors. The reasons are not fully understood. CD4 ${ }^{+}$T-regulatory cells (Tregs) of the Th1-type and follicular helper cells were reported to be associated with favorable prognosis, whereas Th2-type Tregs were demonstrated to inhibit the antitumor immune responses $(33,34)$. The presence of $\mathrm{CD} 8^{+} \mathrm{TILs}$ is significantly correlated with response to therapy and survival of patients (34). While TILs have been investigated by H\&E-staining in lung 

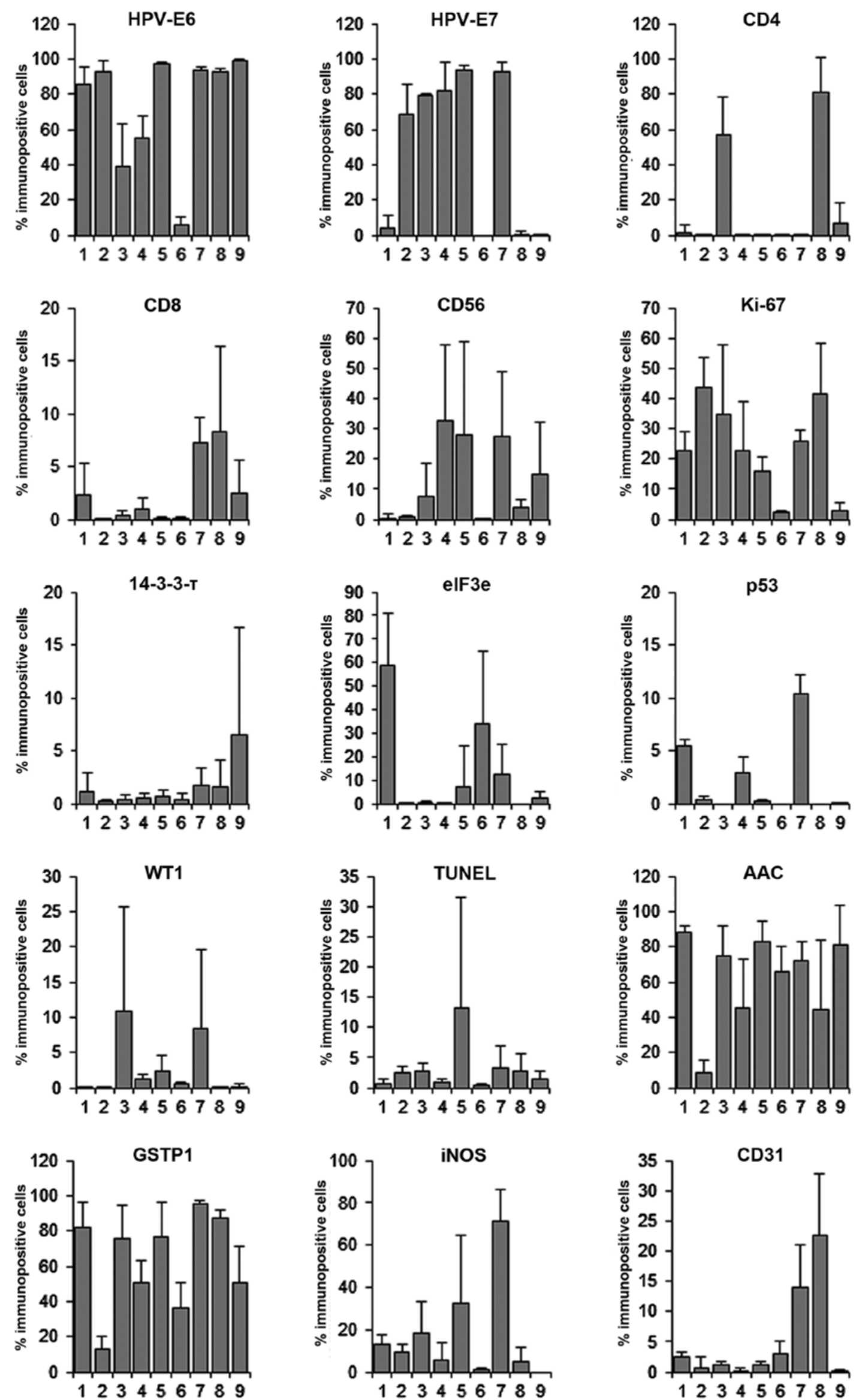

Figure 4. Quantification of immunohistochemical detection of biomarkers by Panoramic Viewer software (3DHISTECH) in nine pediatric nasopharyngeal carcinoma samples. Each six tumor areas free of necrotic and stromal parts were independently quantified per tumor sample. Values are presented as the mean \pm standard deviation. HPV, human papilloma virus; eIF3e, eukaryotic translation initiation factor 3 subunit E; WT1, Wilms tumor 1; TUNEL, terminal deoxynucleotidyl transferase dUTP nick-end labelling; AAC, ATP-ADP translocase; GSTP1, glutathione S-transferase $\pi$ 1; iNOS, inducible nitric oxide synthetase. 
A

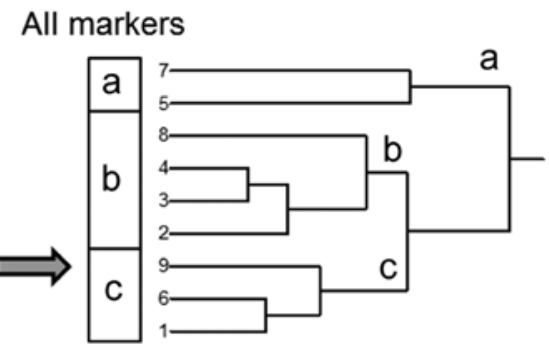

B

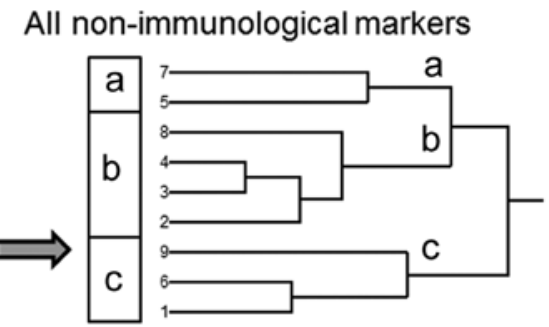

$\mathrm{C}$

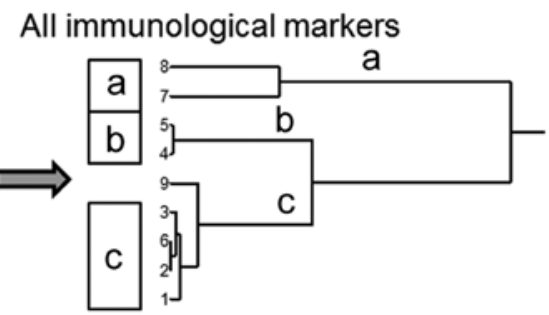

Figure 5. Hierarchical cluster analysis of selected immunohistochemical markers in nine patients with nasopharyngeal carcinoma. Cluster analysis is a distance/proximity-based approach to unravel structures in large data sets. In systems biology, cluster analyses are valuable to define profiles of genes ('gene signatures'), whose expression is linked to biological phenomena, i.e. histological subtypes of tumors, resistance or sensitivity of tumors towards anticancer treatments, survival chances of patients with cancer. Analysis was performed for (A) all markers, (B) all non-immunogenic markers and (C) all immunological markers. The presented dendrograms are based on calculations using the Ward cluster analysis method WinSTAT software.

cancer, breast cancer, laryngeal squamous cell carcinoma and melanoma $(15,36-38)$, to the best of our knowledge, there is no such report on NPC. The present investigation demonstrated, for the first time, that TILs can also be detected by H\&E-staining in NPC. As NPC case 9 contained no TILs in the stromal parts of the tumor, we hypothesize that the patient exhibited immunodeficiency.

Several immunotherapeutic strategies against NPC have been suggested. EBV-latent membrane proteins (LMP1 and LMP2) on the surface of NPC cells may act as targets for $\mathrm{CD}^{+}$ and $\mathrm{CD}^{+} \mathrm{T}$-cells (35). Treatment of NPC using dendritic cells loaded with virus-associated antigens (such as LMP2) elicited antitumor immune responses $(40,41)$. Following stimulation of EBV antigenicity with gemcitabine and valproic acid, treatment of NPC with valganciclovir increased weak antitumor immune responses (36). Bispecific antibodies simultaneously target tumor and T-cell antigens to bring T-cells in close contact with NPC cells (37). From a previous study, a patient with relapsed NPC received salvage adoptive immunotherapy with EBV-specific CTLs, which were reactivated ex vivo from a HLA-identical sibling; a marked increase of endogenous tumor-infiltrating $\mathrm{CD}^{+} \mathrm{T}$-cells was observed, indicating the induction of antitumor effects in the patient (38).
The patient presented here was also refractory to chemo-, radio- and $\beta$-INF therapy, and to allogenic stem cell transplantation. It was hoped that $\mathrm{IgG}$ autoantibodies against EBV-associated antigens of the EBV-infected, but otherwise healthy brother could elicit a T-cell-mediated antiviral response (graft-versus-tumor response) against the tumor of the sister, and that this expected immune response may be transferred with the transplantation. Unfortunately, a sustainable tumor remission was not reached. The patient was severely immunodeficient. The addition of $\beta$-INF to the NPC-2003-GPOH treatment protocol was ineffective, although the beneficial effect of $\beta$-INF for the outcome of patients with NPC has been convincingly demonstrated by several clinical trials $(13,14)$. Another indicator of the immunodeficient state of the patient was the infection with several other viruses and fungi in addition to EBV, including HSV, HHV-6, VZV and Candida. Although immunotherapeutic approaches may be attractive alternatives for non-responders to conventional treatments, immunodeficiency represents a resistance mechanism to immunostimulatory strategies, as indicated by the patient presented in this study.

The rationale to investigate the other biomarkers was their prognostic value for NPC reported in the literature. Although these markers were not able to discriminate case 9 from the other NPC cases, they are valuable markers to describe the aggressiveness of larger cohorts of NPC and other tumor types.

AAC staining was strong in all tumors, whereas, p53 detection was minimal. AAC is a mitochondrial protein that facilitates the exchange of ADP and ATP across the inner mitochondrial membrane and has an essential role in cellular energy metabolism. AAC is involved in metabolic adaptation during tumor development (Warburg effect) (39) causing apoptosis resistance in cancer cells. This supports the observation of low rates of apoptosis in the current study (TUNEL assay). It is not surprising that wild-type p53 was not highly detected, if the high AAC expression and low apoptosis rates are taken into consideration. Wild-type p53 is a nuclear phosphoprotein that triggers apoptosis or cell cycle arrest under cellular stress conditions (40-42). TP53 gene mutations are the most common genetic feature of tumors, identified in $>50 \%$ of tumors $(49,50)$. Loss of p53 function is associated with poor prognosis and drug resistance $(51,52)$.

The HPV-E6 protein expression was high in almost all NPC biopsies investigated. HPV-E6 expression results from the integration HPV genomes into host chromosomes. HPV-E6 binds to p53 and promotes p53 degradation through the ubiquitinproteasome pathway (43) and the p53 levels decrease (44). This observation coincides with the findings in NPC biopsies in the current study. The HPV-E7 protein was expressed in certain patients in the present study. HPV-E7 targets retinoblastomaassociated protein, $\mathrm{pRb}$, which negatively regulates $\mathrm{G} 1 / \mathrm{S}$ and G2/M cell cycle transitions (45).

$\mathrm{Ki}-67$ is expressed during all cell cycle phases (G1, S, $\mathrm{G} 2$ and M) (46). It is a marker of cell proliferation and poor prognosis $(47,48)$. Ki-67 was detected in almost all of our NPC specimens. The clinical significance of Ki-67 in patients with NPC has been reported previously (49).

Eukaryotic translation initiation factors (eIFs) are involved in the initiation of translation. The eIF3 complex binds to 40S ribosomal subunits and promotes the binding of methionyl-tRNA 

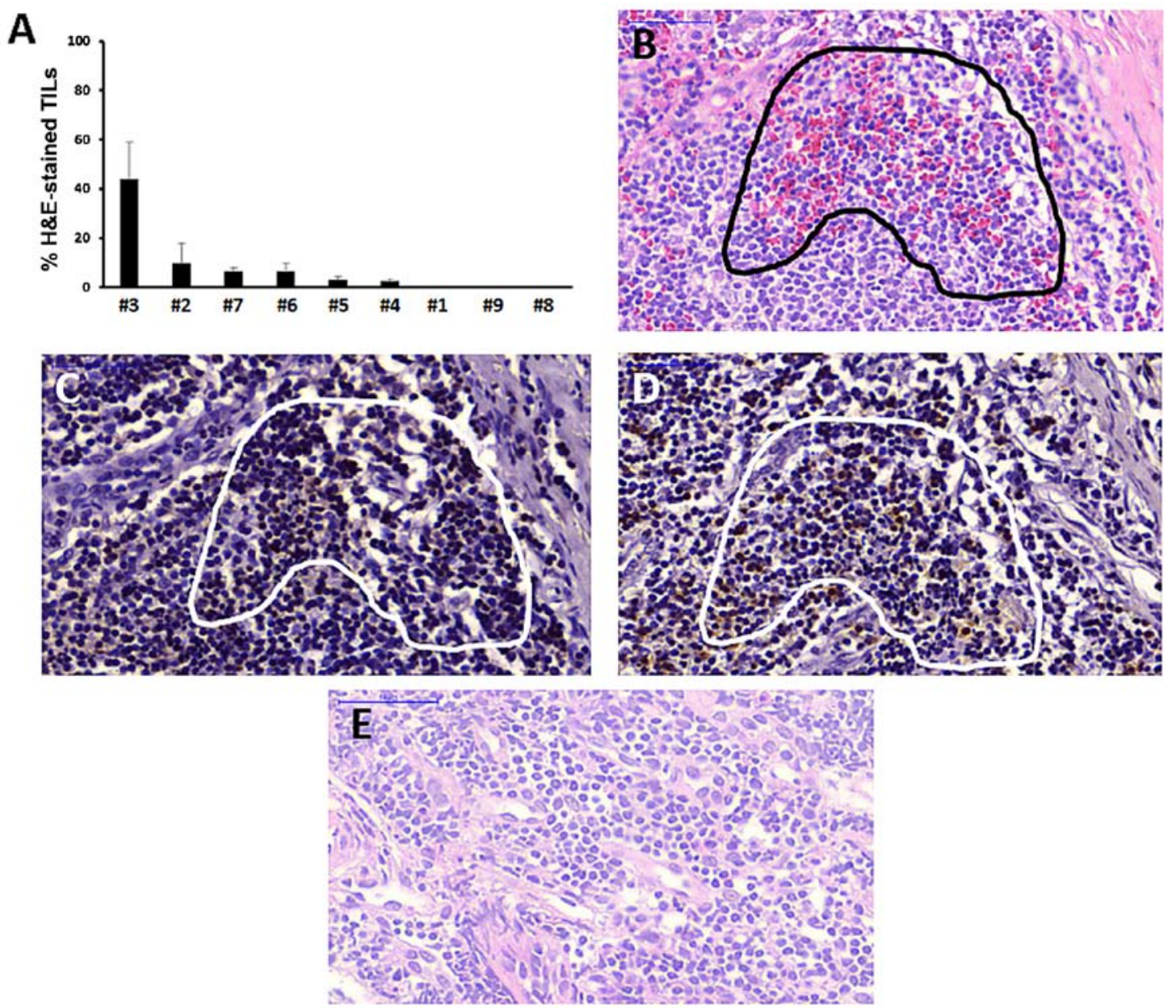

Figure 6. Evaluation of TILs in H\&E-stained sections of nine pediatric NPC. (A) TILs were counted using Panoramic Viewer software (3DHistech) and represented as percentage. Serial sections of NPC case 3 (B) stained by H\&E or immunostained for (C) CD4 or (D) CD8. (E) Case \#9 stained by H\&E. TILs appear red in H\&E-stained sections. TILs, tumor-infiltrating lymphocytes; H\&E, hematoxylin and eosin; NPC, nasopharyngeal carcinoma.

and mRNA to form the $40 \mathrm{~S}$ initiation complex (50). The eIF3a is involved in cell cycle progression (51) and has a role in tumorigenesis, re-sensitization to chemotherapeutics and improved prognosis (52). In the current study, the expression of eIF3e was detected in only $20 \%$ of NPC cases.

Nitric oxide (NO) is an inflammatory mediator and contributes to the inhibition of tumor suppressor functions and DNA repair, activation of oncogenes, angiogenesis, and metastasis (53-55). iNOS generates high amounts of $\mathrm{NO}$ over prolonged periods $(63,64)$. The overexpression of iNOS is associated with high apoptosis rates, whereas low iNOS expression is associated with increased incidences of local tumor recurrence and metastasis following radiotherapy in patients with NPC (56). Tumor-derived iNOS is a pro-angiogenic factor and has been strongly implicated in angiogenesis via upregulation of vascular endothelial growth factor (57). In the present study, iNOS and CD31 (blood vessel marker) were observed in $~ 1 / 3$ of patients.

In conclusion, childhood NPC has an excellent prognosis $(13,14)$. However, as not all children are cured, research efforts have to be undertaken to improve current therapy options. The current study presented a patient with nonkeratinizing NPC type IIb (T2N0M0), that was resistant to chemo-, radio- and $\beta$-IFN therapy, and to allogenic stem cell transplantation. Immunohistochemistry was performed to identify factors distinguishing this patient from a panel of other patients with NPC, who experienced complete remission following conventional therapy. By hierarchical cluster analysis, detection of immunological factors (CD4, CD8 and CD56) separated this patient from the others. The tumor in this patient recurred following $\beta$-IFN therapy, and EBV-directed autoantibodies of the HLA-identical brother did not provoke a graft-versus-tumor response upon allogenic stem cell transplantation. This and several concomitant infections indicated severe immunodeficiency as factor contributing to the fatal outcome. The analysis of more rare cases like this one may help to further improve treatment success of refractory childhood NPC in the future.

\section{Acknowledgements}

We thank Mrs. Doris Rohr (Department of Pharmaceutical Biology, Institute of Pharmacy and Biochemistry, Johannes Gutenberg University, Mainz, Germany) for technical assistance with immunohistochemistry staining.

\section{Funding}

The authors are grateful for the financial support of the Förderkreis Hilfe für krebskranke Kinder Aachen. e.V. 


\section{Availability of data and materials}

The data that support the findings of this study are available from Department of Pediatric and Adolescent Medicine, University Hospital Aachen (Aachen, Germany) and Department of Paediatric Haematology/Oncology, Children's University Hospital (Tübingen, Germany), but restrictions apply to the availability of these data, which were used under patients' consent for the current study, and so are not publicly available. Data are however available from the Department of Pediatric and Adolescent Medicine, University Hospital Aachen, and Department of Paediatric Haematology/Oncology, Children's University Hospital.

\section{Authors' contributions}

TE, RM and RH designed the study; MEMS performed the immunostaining and wrote the manuscript; RM and $\mathrm{RH}$ provide the material and treated the patients; TE performed the statistical analysis, wrote the paper, supervised the work and provided the facilities for the study. All authors read the manuscript and approved the final version.

\section{Ethics approval and consent to participate}

Ethical approval (reference number: EK034/03) for the use of tumor material for experimental purposes was obtained from the Ethics Committee of the University of Aachen (Aachen, Germany) on May 19th, 2003, and local ethical approval was obtained from the participating centers in Germany (14). Written informed consent for experimental work was obtained from all patients.

\section{Consent for publication}

Written informed consent for publication was obtained from all patients.

\section{Competing interests}

The authors declare that they have no competing interests.

\section{References}

1. Parkin DM and Muir CS: Cancer Incidence in Five Continents Comparability and quality of data. IARC Sci Publ 120: 45-173, 1992.

2. Chong VH, Telisinghe PU, Lim E, Abdullah MS, Idris F and Chong CF: Declining incidence of nasopharyngeal carcinoma in brunei darussalam: A three decade study (1986-2014). Asian Pac J Cancer Prev 16: 7097-7101, 2015.

3. Ferlay J, Soerjomataram I, Dikshit R, Eser S, Mathers C, Rebelo M, Parkin DM, Forman D and Bray F: Cancer incidence and mortality worldwide: Sources, methods and major patterns in GLOBOCAN 2012. Int J Cancer 136: E359-E386, 2015.

4. Healy GB: Malignant tumors of the head and neck in children: Diagnosis and treatment. Otolaryngol Clin North Am 13: 483-488, 1980.

5. Cunningham MJ, Myers EN and Bluestone CD: Malignant tumors of the head and neck in children: A twenty-year review. Int J Pediatr Otorhinolaryngol 13: 279-292, 1987.

6. Wang Y,Zhang Y and Ma S: Racial differences in nasopharyngeal carcinoma in the United States. Cancer Epidemiol 37: 793-802, 2013.

7. Chan AT, Teo PM and Johnson PJ: Nasopharyngeal carcinoma. Ann Oncol 13: 1007-1015, 2002.
8. Society AC: Cancer Facts \& Figures 2015. Nasopharyngeal Cancer. American Cancer Society, Atlanta, GA, pp1-44, 2015.

9. Singhi AD, Califano J and Westra WH: High-risk human papillomavirus in nasopharyngeal carcinoma. Head Neck 34: 213-218, 2012.

10. Robinson M, Suh YE, Paleri V, Devlin D, Ayaz B, Pertl L and Thavaraj S: Oncogenic human papillomavirus-associated nasopharyngeal carcinoma: An observational study of correlation with ethnicity, histological subtype and outcome in a UK population. Infect Agent Cancer 8: 30, 2013.

11. Lo EJ, Bell D, Woo J, Li G, Hanna EY, El-Naggar AK and Sturgis EM: Human papillomavirus \& WHO type I nasopharyngeal carcinoma. Laryngoscope 120 (Suppl 4): S185, 2010.

12. Laantri N, Attaleb M, Kandil M, Naji F, Mouttaki T, Dardari R, Belghmi K, Benchakroun N, El Mzibri M and Khyatti M: Human papillomavirus detection in moroccan patients with nasopharyngeal carcinoma. Infect Agent Cancer 6: 3, 2011.

13. Giannoudis A, Ergazaki M, Segas J, Giotakis J, Adamopoulos G, Gorgoulis V and Spandidos DA: Detection of Epstein-Barr virus and human papillomavirus in nasopharyngeal carcinoma by the polymerase chain reaction technique. Cancer Lett 89: 177-181, 1995.

14. Buehrlen M, Zwaan CM, Granzen B, Lassay L, Deutz P, Vorwerk P, Staatz G, Gademann G, Christiansen H, Oldenburger F, et al: Multimodal treatment, including interferon beta, of nasopharyngeal carcinoma in children and young adults: Preliminary results from the prospective, multicenter study NPC-2003-GPOH/DCOG. Cancer 118: 4892-4900, 2012.

15. Kontny U, Franzen S, Behrends U, Bührlen M, Christiansen H, Delecluse H, Eble M, Feuchtinger T, Gademann G, Granzen B, et al: Diagnosis and treatment of nasopharyngeal carcinoma in children and adolescents - Recommendations of the GPOH-NPC Study Group. Klin Padiatr 228: 105-112, 2016.

16. Fischer AH, Jacobson KA, Rose J and Zeller R: Hematoxylin and eosin staining of tissue and cell sections. CSH Protoc 2008: pdb.prot4986, 2008.

17. Salgado R, Denkert C, Demaria S, Sirtaine N, Klauschen F, Pruneri G, Wienert S, Van den Eynden G, Baehner FL, PenaultLlorca F, et al; International TILs Working Group 2014: The evaluation of tumor-infiltrating lymphocytes (TILs) in breast cancer: Recommendations by an International TILs Working Group 2014. Ann Oncol 26: 259-271, 2015.

18. Kadioglu O and Efferth T: Pharmacogenomic characterization of cytotoxic compounds from Salvia officinalis in cancer cells. J Nat Prod 78: 762-775, 2015.

19. Schielke HJ, Fishman JL, Osatuke K and Stiles WB: Creative consensus on interpretations of qualitative data: The Ward method. Psychother Res 19: 558-565, 2009.

20. Efferth T, Fabry U and Osieka R: Apoptosis and resistance to daunorubicin in human leukemic cells. Leukemia 11: 1180-1186, 1997.

21. Scherf U, Ross DT, Waltham M, Smith LH, Lee JK, Tanabe L, Kohn KW, Reinhold WC, Myers TG, Andrews DT, et al: A gene expression database for the molecular pharmacology of cancer. Nat Genet 24: 236-244, 2000.

22. Mocellin S, Provenzano M, Rossi CR, Pilati P, Nitti D and Lise M: DNA array-based gene profiling: From surgical specimen to the molecular portrait of cancer. Ann Surg 241: 16-26, 2005.

23. Eisen MB, Spellman PT, Brown PO and Botstein D: Cluster analysis and display of genome-wide expression patterns. Proc Natl Acad Sci USA 95: 14863-14868, 1998.

24. Perou CM, Sørlie T, Eisen MB, van de Rijn M, Jeffrey SS, Rees CA, Pollack JR, Ross DT, Johnsen H, Akslen LA, et al: Molecular portraits of human breast tumours. Nature 406: 747-752, 2000

25. Ross DT, Scherf U, Eisen MB, Perou CM, Rees C, Spellman P, Iyer V, Jeffrey SS, Van de Rijn M, Waltham M, et al: Systematic variation in gene expression patterns in human cancer cell lines. Nat Genet 24: 227-235, 2000.

26. Yang J, Zhou M, Zhao R, Peng S, Luo Z, Li X, Cao L, Tang K, Ma J, Xiong W, et al: Identification of candidate biomarkers for the early detection of nasopharyngeal carcinoma by quantitative proteomic analysis. J Proteomics 109: 162-175, 2014.

27. Cheuk DK, Billups CA, Martin MG, Roland CR, Ribeiro RC, Krasin MJ and Rodriguez-Galindo C: Prognostic factors and long-term outcomes of childhood nasopharyngeal carcinoma. Cancer 117: 197-206, 2011.

28. Volm M, Koomägi R, Mattern J and Efferth T: Expression profile of genes in non-small cell lung carcinomas from long-term surviving patients. Clin Cancer Res 8: 1843-1848, 2002. 
29. Volm M, Koomägi R, Mattern J and Efferth T: Protein expression profiles indicative for drug resistance of non-small cell lung cancer. Br J Cancer 87: 251-257, 2002.

30. Volm M, Koomägi R, Mattern J and Efferth T: Protein expression profile of primary human squamous cell lung carcinomas indicative of the incidence of metastases. Clin Exp Metastasis 19: 385-390, 2002

31. Trempat P, Tabiasco J, Andre P, Faumont N, Meggetto F, Delsol G, Gascoyne RD, Fournie JJ, Vivier E and Brousset P: Evidence for early infection of nonneoplastic natural killer cells by Epstein-Barr virus. J Virol 76: 11139-11142, 2002.

32. Yuling H, Ruijing X, Li L, Xiang J, Rui Z, Yujuan W, Lijun Z, Chunxian D, Xinti T, Wei X, et al: EBV-induced human CD8 ${ }^{+}$ NKT cells suppress tumorigenesis by EBV-associated malignancies. Cancer Res 69: 7935-7944, 2009.

33. Zheng Y, Cao KY, Ng SP, Chua DT, Sham JS, Kwong DL, Ng MH $\mathrm{Lu} \mathrm{L}$ and Zheng BJ: Complementary activation of peripheral natural killer cell immunity in nasopharyngeal carcinoma. Cancer Sci 97: 912-919, 2006.

34. Mahmoud SM, Paish EC, Powe DG, Macmillan RD, Grainge MJ, Lee AH, Ellis IO and Green AR: Tumor-infiltrating CD8 ${ }^{+}$ lymphocytes predict clinical outcome in breast cancer. J Clin Oncol 29: 1949-1955, 2011

35. Haigh TA, Lin X, Jia H, Hui EP, Chan AT, Rickinson AB and Taylor GS: EBV latent membrane proteins (LMPs) 1 and 2 as immunotherapeutic targets: LMP-specific $\mathrm{CD}^{+}{ }^{+}$cytotoxic $\mathrm{T}$ cell recognition of EBV-transformed B cell lines. J Immunol 180 : $1643-1654,2008$

36. Stoker SD, Novalić Z, Wildeman MA, Huitema AD, Verkuijlen SA Juwana H, Greijer AE, Tan IB, Middeldorp JM and de Boer JP: Epstein-Barr virus-targeted therapy in nasopharyngeal carcinoma. J Cancer Res Clin Oncol 141: 1845-1857, 2015.

37. Taylor GS, Haigh TA, Gudgeon NH, Phelps RJ, Lee SP, Steven NM and Rickinson AB: Dual stimulation of Epstein-Barr Virus (EBV)-specific $\mathrm{CD} 4^{+}-$and $\mathrm{CD} 8^{+}-\mathrm{T}$-cell responses by a chimeric antigen construct: Potential therapeutic vaccine for EBV-positive nasopharyngeal carcinoma. J Virol 78: 768-778 2004

38. Comoli P, De Palma R, Siena S, Nocera A, Basso S, Del Galdo F, Schiavo R, Carminati O, Tagliamacco A, Abbate GF, et al: Adoptive transfer of allogeneic Epstein-Barr virus (EBV)-specific cytotoxic $\mathrm{T}$ cells with in vitro antitumor activity boosts LMP2-specific immune response in a patient with EBV-related nasopharyngeal carcinoma. Ann Oncol 15: 113-117, 2004.

39. Chevrollier A, Loiseau D, Reynier P and Stepien G: Adenine nucleotide translocase 2 is a key mitochondrial protein in cancer metabolism. Biochim Biophys Acta 1807: 562-567, 2011.

40. Wang Z and Sun Y: Targeting p53 for novel anticancer therapy. Transl Oncol 3: 1-12, 2010.

41. Vogelstein B, Lane D and Levine AJ: Surfing the p53 network. Nature 408: 307-310, 2000
42. Giaccia AJ and Kastan MB: The complexity of p53 modulation: Emerging patterns from divergent signals. Genes Dev 12: 2973-2983, 1998.

43. Scheffner M, Werness BA, Huibregtse JM, Levine AJ and Howley PM: The E6 oncoprotein encoded by human papillomavirus types 16 and 18 promotes the degradation of $\mathrm{p} 53$. Cell 63: 1129-1136, 1990.

44. Matlashewski G, Banks L, Wu-Liao J, Spence P, Pim D and Crawford L: The expression of human papillomavirus type 18 E6 protein in bacteria and the production of anti-E6 antibodies. J Gen Virol 67: 1909-1916, 1986.

45. Yim EK and Park JS: The role of HPV E6 and E7 oncoproteins in HPV-associated cervical carcinogenesis. Cancer Res Treat 37 319-324, 2005

46. Kim KH and Sederstrom JM: Assaying cell cycle status using flow cytometry. Curr Protoc Mol Biol 111: 28.6.1-11, 2015.

47. Urruticoechea A, Smith IE and Dowsett M: Proliferation marker Ki-67 in early breast cancer. J Clin Oncol 23: 7212-7220, 2005.

48. Liu P, Sun YL, Du J, Hou XS and Meng H: CD105/Ki67 coexpression correlates with tumor progression and poor prognosis in epithelial ovarian cancer. Int J Gynecol Cancer 22: 586-592, 2012

49. Masuda M, Shinokuma A, Hirakawa N, Nakashima T and Komiyama S: Expression of bcl-2-, p53, and Ki-67 and outcome of patients with primary nasopharyngeal carcinomas following DNA-damaging treatment. Head Neck 20: 640-644, 1998.

50. Hershey JW: The role of eIF3 and its individual subunits in cancer. Biochim Biophys Acta 1849: 792-800, 2015.

51. Dong Z, Liu Z, Cui P, Pincheira R, Yang Y, Liu J and Zhang JT: Role of eIF3a in regulating cell cycle progression. Exp Cell Res 315: 1889-1894, 2009.

52. Yin JY, Shen J, Dong ZZ, Huang Q, Zhong MZ, Feng DY, Zhou HH, Zhang JT and Liu ZQ: Effect of eIF3a on response of lung cancer patients to platinum-based chemotherapy by regulating DNA repair. Clin Cancer Res 17: 4600-4609, 2011

53. Lala PK and Orucevic A: Role of nitric oxide in tumor progression: Lessons from experimental tumors. Cancer Metastasis Rev 17: 91-106, 1998.

54. Wink DA, Ridnour LA, Hussain SP and Harris CC: The reemergence of nitric oxide and cancer. Nitric Oxide 19: 65-67, 2008.

55. Beckman JS, Beckman TW, Chen J, Marshall PA and Freeman BA: Apparent hydroxyl radical production by peroxynitrite: Implications for endothelial injury from nitric oxide and superoxide. Proc Natl Acad Sci USA 87: 1620-1624, 1990

56. Jayasurya A, Dheen ST, Yap WM, Tan NG, Ng YK and Bay BH: Inducible nitric oxide synthase and bcl-2 expression in nasopharyngeal cancer: Correlation with outcome of patients after radiotherapy. Int J Radiat Oncol Biol Phys 56: 837-845, 2003.

57. Kane AJ, Barker JE, Mitchell GM, Theile DR, Romero R, Messina A, Wagh M, Fraulin FO, Morrison WA and Stewart AG: Inducible nitric oxide synthase (iNOS) activity promotes ischaemic skin flap survival. Br J Pharmacol 132: 1631-1638, 2001. 